EMBRYARIDDLE
Aeronautical University

SCHOLARLY COMMONS
International Journal of Aviation, Aeronautics, and Aerospace

\title{
Integrating Fatigue Management with Safety Management Systems for Commercial Flightcrew Operations
}

Arnold W. Starr Jr.

Embry-Riddle Aeronautical University, starrjra@my.erau.edu

Follow this and additional works at: https://commons.erau.edu/ijaaa

Part of the Management and Operations Commons

\section{Scholarly Commons Citation}

Starr, A. W. (2017). Integrating Fatigue Management with Safety Management Systems for Commercial Flightcrew Operations. International Journal of Aviation, Aeronautics, and Aerospace, 4(1).

https://doi.org/10.15394/ijaaa.2017.1143

This Literature Review is brought to you for free and open access by the Journals at Scholarly Commons. It has been accepted for inclusion in International Journal of Aviation, Aeronautics, and Aerospace by an authorized administrator of Scholarly Commons. For more information, please contact commons@erau.edu. 
Fatigue is a well-studied topic in aviation. As in many industries, aviation service providers must balance the hazards of fatigue in the workplace along with other risks to provide a product the consumer values and has confidence in, ensure a safe work environment, and protect resources. Aviation does have unique risks, especially when considered cumulatively: complex machines, harsh operating environments, regulated long work periods that cover a 24-hour, 7-day a week cycle, and extended periods of inactivity for flight crew members sandwiched in between relatively brief, high-intensity workloads. These factors, which are often in flux, make managing fatigue difficult for all stakeholders. The Federal Aviation Administration (FAA) made significant rule changes to reduce risk exposure due to fatigue by reducing the amount of flight time allowed for pilots to fly, increasing the required time off between flight duty periods, and mandating fatigue management training for flight crews (FAA, 2011). However, certain elements, such as fitness for duty and responsibility for commuting, while specifically addressed and defined in the regulatory documents, are largely left up to individual flight crew member determination.

The FAA has also implemented requirements for certificate holders to develop a Safety Management System (SMS) within their organizations (FAA, 2015) which, among other fundamentals, advocates the establishment of strong safety culture. This includes both a reporting and just culture to encourage the reporting of safety issues without the threat of retaliation (Stolzer \& Goglia, 2015). A significant element of the FAA's prescriptive flight duty rules and requirements is the obligation of flight crews to manage and account for their fatigue and fitness for duty. The certificate holder has an obligation to facilitate this as well, starting with a safety policy that includes a commitment to fatigue management. It is within the framework of a functioning SMS that the challenges of managing fatigue and the hazards produced by it need to be addressed.

This article explores the current state of fatigue management in the industry and the role the current prescriptive structure of the regulatory environment is having on reducing the hazards associated with fatigue. Included is a brief review of the National Transportation Safety Board (NTSB) reports on two commercial aviation accidents where fatigue was indicated as a factor. Finally, it will highlight the benefits of using a Fatigue Risk Management System (FRMS), within the framework of the organization's functioning SMS, to conduct research and data collection to ensure flight crew members are receiving the rest intended by current regulation. 


\section{Rules, Regulations, and Advisories}

\section{Part 117}

Following testimony in the US Congress regarding fatigue in commercial aviation, the FAA instituted a thorough process to amend its flight, duty, and rest rules under 14 Code of Federal Register (CFR) Parts 117,119, and 121 Flight Crew Member Duty and Rest Requirements (referred to in the industry as "Part 117"). This was published in January 2012, with an effective date two years later of January 14, 2014. These supplanted rules that had been in place nearly 70 years. With Part 117, the FAA took a systems approach to fatigue management, acknowledging that mitigating the risks that fatigue poses to passenger travel will need both the carrier and flight crew member to accept responsibility. Part 117 is very detailed, prescriptive, and incorporated into the Federal Register. Included in the document is an educational primer on fatigue, examples of accidents caused in part by fatigue, a history of recommendations made by the NTSB, and input from various stakeholders throughout the rulemaking process. The rule includes tables detailing work/rest rules that list start times, the number of flight segments and consecutive days worked, attempting to prescriptively manage fatigue based upon these objective factors. Included is a clarification on the requirement for regular education of flight crew members on the effects of fatigue, available mitigating strategies, and the importance of being fit for duty. Also, as referenced in the rule, the FAA has promulgated numerous circulars, articles, and newsletters concerning the effects and management of fatigue and various education programs and tools for service providers. Most of these are either advisory or voluntary and offer a wealth of information to assist both flight crew members and operators not only to comply with the regulation but more importantly, understand the challenges that fatigue presents. Two of these warrant a closer look: Advisory Circular (AC) 120103A, Fatigue Risk Management Systems, and AC 117-3, Fitness for Duty.

\section{Fatigue Risk Management Systems}

The objectives of an FRMS, as advocated by the FAA, are to "manage, monitor, and mitigate the effects of fatigue to improve flight crew member alertness and reduce performance errors" as well as to balance safety and productivity (FAA, 2013). FRMS is a voluntary program, and the circular describes itself as an acceptable means of implementation. The AC lists four tools: data collection, analysis, identification of causes, and application of procedures. The key to this process is the collection and usage of existing data collection systems. These include Flight Operational Quality Assurance (FOQA), the Aviation Safety Action Program (ASAP), and the Aviation Safety Reporting System (ASRS). The FAA 
recommends this information be analyzed, for example, by using a Root Cause Analysis (RCA) to determine if the events described in the report can be attributed to fatigue. Also emphasized is the need to have an open and just reporting system, intrinsic to SMS principles, to ensure this information remains attainable. Correspondingly, listed in the document is assignment of roles and responsibilities of both the certificate holder and individual employees. Similar to fundamentals of an SMS, the FRMS is data driven and based upon valid scientific principles. The FRMS allows certificate holders more flexibility and complements the prescriptive regulations on flight duty and rest requirements (FAA, 2013).

Fitness for Duty Worthy of examination as it pertains to the thesis of this article is AC 117-3, Fitness for Duty. Drawing from Part 117, this AC defines fit for duty as "being physiologically and mentally prepared and capable of performing the assigned duties at the highest degree of safety" (Part 117, Section 117.3). The purpose of this AC is to help certificate holders and flight crew members understand their responsibilities under Part 117 and acceptable methods of compliance. It specifically targets commuting, and guides educate flight crew members on the potential effects of commuting on fatigue. The emphasis on commuting is noteworthy, as this aspect of commercial aviation is largely unregulated. The AC emphasized that fitness for duty be a joint responsibility between flight crew members and the certificate holder.

\section{Fatigue in Commercial Aviation}

The effects of fatigue have been studied for decades, and the hazards induced by fatigue are well documented in both scientific studies and accident investigations. The NTSB has cited fatigue as a contributing factor in multiple investigations, and as far back as 1972, expressed concerns about the effects of fatigue, sleep and circadian effects on performance (FAA, 2011). While fatigue can be a hazard in numerous professions, as previously indicated it presents some unique challenges in aviation. Many are intrinsic to the industry however fatigue may be to a degree self-induced by choices made by the individuals themselves due to the liberal travel privileges many flight crew members in the commercial aviation industry possess.

Mallis, Banks and Dinges begin their discussion of fatigue with the classical definition, "a decrease in performance capability as a function of time on task" and further stress that this formed the basis for FAA duty time regulations for over 70 years (2010, P. 401). There exist more current and scientific definitions, and all share common themes. These can relate to the manner in which it was accumulated. For example, acute fatigue is related to the effects with short-term duty. This 
contrasts with chronic fatigue, which is associated with consecutive work periods whereby insufficient rest causes the effects to mount up (Nunes \& Cabon, 2015). The FAA describes it in a similar form, labeling fatigue as transient (acute), cumulative (chronic), and adds category labeled circadian. This last classification describes reduced performance typically during the hours of 2 a.m. to 6 a.m., referred to as WOCL, for Window of Circadian Low (FAA, 2011). Also described in this FAA document and, just as important to the discussion, are the effects of fatigue. These include attention lapses, reduced performance, delayed reactions, impaired decision making, reduced situational awareness, and lowered drive to perform elective tasks.

A review of the literature reveals significant research in fatigue and fatigue management. Fatigue is recognized as a threat that affects alertness and cognitive performance; it is evident from experience that humans are simply not constructed to operate efficiently on around-the-clock schedules (Mallis, Banks \& Dinges, 2010). Several research driven models of fatigue management have been proposed and studied. Dr. Rosekind and his associates at Alertness Solutions of Cupertino, CA developed and studied a comprehensive program labeled Alertness Management Program (AMP) that included education, scheduling, alertness strategies, and the promotion of healthy sleep (Rosekind, Gregory \& Mallis, 2006). The goal of AMP is to apply scientific data to actual policies and improve scheduling practices. Another model is SAFTE, for Sleep, Activity, Fatigue, and Task Effectiveness. This model combines sleep/wake history with daily circadian cycles to produce a "percent effectiveness" scale (Rangan \& Van Dongen, 2013). The concept behind this model is to determine the threshold for an acceptable level of fatigue and apply that to duty scheduling in commercial aviation.

The most current approach endorsed by the FAA is the FRMS, as was introduced above (FAA, 2013). The FAA recognizes the value of these systems by allowing companies with an approved FRMS to deviate from the prescriptive requirements for flight duty management and have more latitude to meet regulatory requirements. Two challenges to implementing FRMS are commonly identified: determining which types of data are to be monitored and the methods to do that, and how to measure the effectiveness of FRMS in managing risk (Gander, Mangie, Van Den Berg, Smith, Mulrine \& Signal, 2014). Similar to modern safety management systems, FRMS is necessarily data driven. However, unlike routinely collected flight data, for example, FOQA, monitoring and recording measurements of crew fatigue can require considerable resources (Gander et al., 2014). Voluntary reporting systems as explained in the accepted descriptions of SMS can be effective tools in monitoring the FRMS, as can auditing regimens such as the Line Operational Safety Audit (LOSA). 


\section{Colgan Air 3407 \& UPS 1354}

Two serious aviation accidents juxtapose the FAA's issuance of the new Part 117 Flightcrew Member Duty and Rest Requirements and are illustrative of the complex issues surrounding the management of fatigue: Colgan Air 3407 (2009) and UPS 1354 (2011). Focusing first on Colgan Air, the NTSB listed as the probable cause the captain's inappropriate response to a stall warning during a landing approach in icing conditions (NTSB, 2011). The report detailed several issues discovered regarding training practices and adherence to Crew Resource Management (CRM) principles. What is most significant about Colgan Air 3407, however, is it became the catalyst to reopen discussion/debate concerning the regulations on aircrew member duty time and rest requirements, reaching all the way to the floor of Congress (Arnoult, 2009). This was due in large part from the commuting habits of the flight crew revealed during the investigation.

There were four items listed in the report's findings that related to fatigue. None were listed as a contributory cause of the accident. The report states that: 1 . Fatigue likely impaired this crew's performance, 2. This crew had a responsibility to manage their work/rest schedule and did not do so, 3. Colgan Air did not "proactively address pilot fatigue hazards" at a hub that is associated with commuting pilots, and 4. Operators like Colgan Air have a responsibility to do so. Following testimony in Congress, the FAA initiated the process to update and amend 14 CFR Parts 117, 119, and 121 Flight Crew Member Duty and Rest Requirements (referred to in the industry as "Part 117"). This was published January 4, 2014.

The crash of United Parcel Service UPS 1354 also revealed systemic issues with training, dispatch, serious breakdowns in CRM, and failure to comply with company Standard Operating Procedures (SOP). Significant in the NTSB report, however, is the board's determination that the first officer's fatigue was contributory and was due to her "ineffective off-duty time management" (NTSB, 2013). The report noted that while UPS, as an all-cargo carrier, was not required to comply with Part 117, the schedule the crew was flying met those requirements. It was discovered during the investigation that the first officer engaged in personal travel during a layover and did not get adequate rest. Furthermore, it appears she was aware of her fatigued state but elected not to comply with company fatigue policy and procedures.

The reluctance of the first officer to call in fatigued was investigated by the board. It was noted that UPS has a flight duty/crew rest policy that is more stringent 
than required by rule and is defined in a collective bargaining agreement between the company and the pilot's union, the International Pilot's Association (IPA). The agreement requires that crew members who call in fatigued have an obligation to submit a report detailing the circumstances that prevented them from getting the proper rest before duty. If it is determined to be the crew member's fault, they would be charged sick time and the event noted in the crew member's file. An IPA representative cited in the report believed this to be punitive in nature. This, of course, is in direct conflict with the core tenants of SMS. To that end, the board recommended to both UPS and the IPA to perform a review of the process used to report fatigue and its effectiveness as a non-punitive method to address fatigue.

\section{SMS and Managing Fatigue}

The application of SMS to fatigue management is fundamental; fatigue is a recognized hazard in aviation and managing the risk it creates is basic to maintaining a sound SMS. This requires that the foundational elements of the SMS be first established. Starting with Safety Policy, the company, through its senior management structure, must make clear the policy and procedures to be followed and the accountability of each member of the organization to comply. This is particularly important with regards to fatigue since the management of it to a great part requires flight crew member self-identification and reporting. Whereas the prescriptive duty rules of Part 117 are well defined, regarding defining fatigue, the FAA "simply chose not to impose a mandatory regulatory requirement because the signs used to identify fatigue cannot be synthesized into a general objective standard" (Part 117, p. 349). Thus, company Safety Policy, as defined by the SMS, must ensure that the standards expected are understood and complied with.

As explained in the AC, not all certificate holders may have the need to implement an FRMS due to various operational factors. However, all are required to submit a Fatigue Risk Management Plan (FRMP) consisting of:

- senior-level management commitment

- fatigue management policies and procedures

- flight time, duty period, and rest plan

- a fatigue reporting program

- an education and awareness training program;

- system for monitoring flight crew fatigue and evaluating the FRMP (Council, N.R., 2012)

An FRMS and an FRMP is a method to manage and mitigate risk through the company's existing safety management process (FAA, 2013). 
Incorporation of an FRMS works in parallel with the elements of the Safety Risk Management component of the organization's SMS. As described in the FAA circular, the FRMS, as a data-driven process, can be a stand-alone system or be incorporated into the organization's existing SMS. As with other hazards in the industry, employing risk management tools can identify and subsequently eliminate/mitigate/control risks. Specifically, for fatigue, these can include strategic crew duty and rest period scheduling based upon actual scientifically validated results.

Likewise, the FRMS supports the Safety Assurance component of SMS by providing, among other things, performance monitoring and a system of employee reporting. As explained by Stolzer \& Goglia, this includes a management review and a plan to incorporate what is learned into current operations to reduce risk and ensure continuous improvement (2015). Safety Assurance relies on documentation and encourages participation so that personnel will not fear retribution for reporting deviations. A key element of this process, as explained in the AC, is the need for the organization to distinguish between unintentional human error that may have been caused by fatigue versus intentional non-compliance with rules, SOP, and the need for a separate system from FRMS to consider deliberate violations (FAA, 2013).

Finally, the fourth pillar of SMS, Safety Promotion, needs to establish safety as a core value with a sustained Safety Culture. As was discovered in the Colgan Air and UPS crashes, a breakdown in the safety culture at multiple levels created a situation where poor decisions were made that were not trapped. Safety Promotion provides the tools to promote safety with training, communication, and awareness (Stolzer \& Goglia, 2015). A fatigue Education and Awareness Training Program is mandated in Part 117 and a required element of an approved FRMS. Figure 1 shows the relationship of SMS to an FRMS. Ultimately, the level at which it occurred is important, but more important is what needs to be done to correct it. 


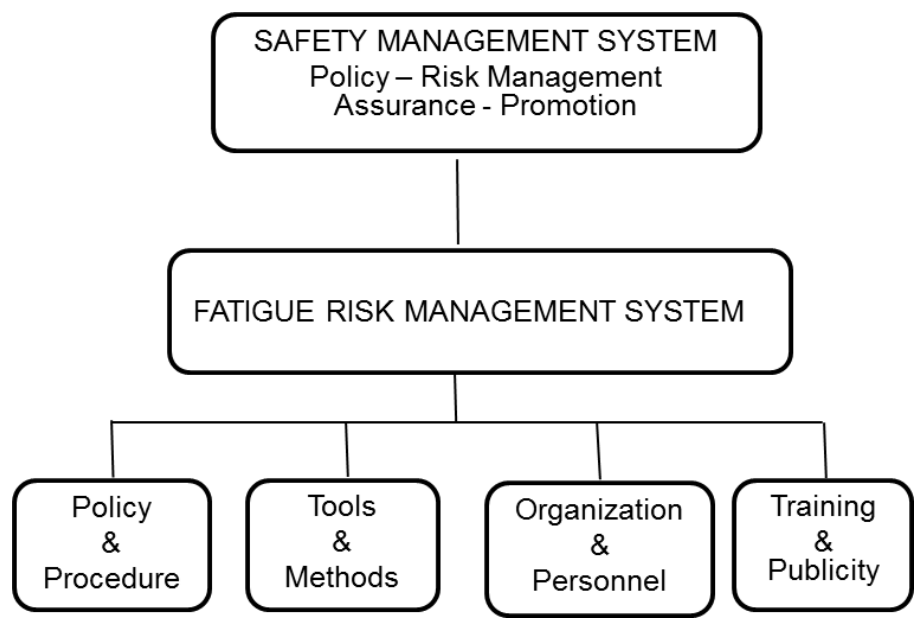

Figure 1. SMS and FRMS

\section{Solutions}

Commercial aviation presents a wide variety of challenges and opportunities to consider when developing a fatigue management strategy. Commercial pilots can traverse multiple time zones and often need to perform for exceptionally long periods of many days (FAA, 2011). On the other hand, commercial pilots often have larger breaks between work cycles, enabling restorative sleep periods not necessarily afforded in other professions. All of these factors must be considered in the development and deployment of an FRMS. However, to be effective, the FRMS needs valid and reliable data.

The establishment of an SMS requires sound and comprehensive safety policies and practices. Violations of these polices can and have led to tragic consequences. Fatigue, although intrinsic to both domestic and worldwide aviation operations, is manageable provided that the hazards are evaluated in total and the risks identified. The FRMS must actively pursue data, analyze the data, and provide solutions to both management and line flight crew members to better understand and control fatigue. This can be done using data generated through existing reporting systems. FOQA, ASAP, and LOSA are good examples. Comparing reported deviations of standard operating procedures or air traffic control clearances against factors such as time of day, time on duty, cumulative work days, etc., may yield correlations useful in an FRMS. FRMS should also employ specific methodology as described in the AMP and SAFTE programs. Gander et al. suggest developing performance indicators from routine scheduling data to monitor the impact of schedule changes on risk assessment and target the causes of fatigue risk, i.e., early departures coupled with extended duty days (2014). 
Other solutions would be to require commuting factors to be included in the calculation of duty time. As discussed in a National Academy of Sciences (NAS) report titled The Effects of Commuting on Pilot Fatigue, this method exists in at least one known collective bargaining agreement (Council N.R., 2012). Finally, further increasing the use of automation may provide some relief in the future, but it does not negate the need for humans in safety-critical industries (Nunes \& Cabon, 2015).

\section{Pilot Perceptions}

In March/April 2014, a team of researchers from Purdue University conducted a survey of commercial pilots on their perceptions of the recently enacted Part 117 (Rudari, Johnson, Geske \& Sperlak, 2016). The team developed a Likert-scale survey asking questions on the impact of the new rules on alertness, fatigue levels, sleep cycles, aircraft operation and overall safety. Also included in the survey instrument was a comment section that allowed for additional, openended input. The survey had a total of 92 participants.

The results from the Likert section was mixed, with many of the respondents indicating neither a positive nor negative impact. The researchers suggest this may be attributed to the relative newness of the rule. The open-ended section, on the other hand, was completed by approximately one-half of the respondents and clearly indicated a negative perception among these pilots, particularly concerning the cargo carrier exclusion and crew rest issues regarding long haul operations. As stated in the study, the overall conclusions may be biased since the survey was conducted within months of the rule being put into effect. However, the comments and concerns reported by pilots in the open-ended section could be useful in future research efforts on this subject (Rudari et al., 2016).

\section{Summary}

Part 117 is clear that fatigue management is a combined responsibility of the certificate holder and the flight crew member. Compliance with the detailed and prescriptive elements of the CFR and related ACs about flight, duty day and rest rules do not relieve any of the affected parties of their obligations to ensure all personnel is fit for duty. The NAS study was sponsored by the FAA and is a thorough examination of fatigue in the aviation community in general, with a focus on the nature of commuting unique to the industry. It found a wide variety of policies and procedures, due in part, to the unregulated aspect of commuting. Many are based upon collective bargaining agreements. However, the report concluded that "no systemic, reliable information was available to the committee about the effects, if any, of commuting pilots, reliably arriving at their domicile on time for duty or about the effects, if any, on either fatigue or fatigue calls" (Council, N.R., 
2012. p. 44). Crewmember insights are an essential element of fatigue management and must be included in working this problem.

Thus, research needs to continue on fatigue and airline scheduling practices using data from all available sources. This includes commuting habits, rest opportunities, and their potential effect on crew fitness and compliance with the intent and goals of Part 117. With this information, procedures, policies, and practices can be developed and implemented to improve fatigue management. An adequate rest facilities at major hubs or compensation incentives to report to the domicile with enough time to secure appropriate rest before scheduled report time should be considered, for example. Ultimately, the FRMS, functioning as a component of the organization's SMS, will yield and maintain a safety culture whereby operating aircraft in a fatigued state, whether from the crewmember's domicile or an en route location, is recognized and treated as unacceptable by all stakeholders in the organization. 


\section{References}

Arnoult, S. (2009). The problem that won't go away. Air Transport World, 46(8), 26.

Council, N. R. (2012). Effects of commuting on pilot fatigue. Washington, DC: National Academies Press.

Federal Aviation Administration (FAA). (2011). Flightcrew member duty and rest requirements. (FAA-2009-1093). Retrieved from https://www.faa.gov/regulations_policies/rulemaking/recently_published/ media/2120-AJ58-FinalRule.pdf

Federal Aviation Administration (FAA) (2013). Fatigue risk management systems (FAA AC 120-103A). Retrieved from http://www.faa.gov/documentLibrary/media/Advisory_Circular/AC_120103A.pdf

Federal Aviation Administration (FAA). (2015). Safety management systems for aviation service providers. (FAA AC 120-92B). Retrieved from http://www.faa.gov/documentLibrary/media/Advisory_Circular/AC_12092B.pdf

Gander, P. H., Mangie, J., Van Den Berg, M. J., Smith, A. T., Mulrine, H. M., \& Signal, T. L. (2014). Crew fatigue safety performance indicators for fatigue risk management systems. Aviation, Space, and Environmental Medicine, 85(2), 139.

Mallis, M., Banks, S. \& Dinges, D. (2010). Aircrew fatigue, sleep need and circadian rhythmicity. In Salas, E., \& Maurino, D. E. (Eds.), Human Factors in Aviation (2nd ed. pp. 402-431). Boston, MA: Academic Press/Elsevier.

Maurino, D. E. \& Salas, E., (Eds.) (2010). Human factors in aviation (Second ed.). Boston, MA: Academic Press/Elsevier.

National Transportation Safety Board (NTSB). (2009). Loss of Control on Approach Colgan Air, Inc. (NTSB/AAR-10/01). Retrieved from http://www.ntsb.gov/investigations/AccidentReports/Reports/AAR1001.p df 
National Transportation Safety Board (NTSB). (2013). Crash during a nighttime nonprecision instrument approach to landing UPS Flight 1354. (NTSB/AAR-14/02). Retrieved from http://www.ntsb.gov/investigations/AccidentReports/Reports/AAR1402.p df

Nunes, A., \& Cabon, P. (2015). The fatigue conundrum. American Scientist, 103(3), 218. doi:10.1511/2015.114.218

Rangan, S., \& Van Dongen, Hans P A. (2013). Quantifying fatigue risk in modelbased fatigue risk management. Aviation, Space, and Environmental Medicine, 84(2), 155.

Rosekind, M. R., Gregory, K. B., \& Mallis, M. M. (2006). Alertness management in aviation operations: Enhancing performance and sleep. Aviation, Space, and Environmental Medicine, 77(12), 1256-1265. doi:10.3357/ASEM.1879.2006

Rudari, L., Johnson, M. E., Geske, R. C., \& Sperlak, L. A. (2016). Pilot Perceptions on Impact of Crew Rest Regulations on Safety and Fatigue. International Journal of Aviation, Aeronautics, and Aerospace, 3(1). http://doi.org/10.15394/ijaaa.2016.1096

Stolzer, A. J., \& Goglia, J. J. (2015). Safety management systems in aviation $\left(2^{\text {nd }}\right.$ ed.). Farnham, Surrey, England: Ashgate.

Stolzer, A. J., Halford, C. D. \& Goglia, J. J. (2011). Implementing safety management systems in aviation. Farnham, Surrey, England: Ashgate. 